\title{
Capsicum-extract blended chitosan composite films and studying their antibacterial properties
}

\author{
SANUJA SRITHAR* and SOWMYA SRINIVASA RAO \\ Department of Chemistry, School of Basic Sciences, Vels Institute of Science, Technology and Advanced Studies, \\ Pallavaram, Chennai 600117, India \\ *Author for correspondence (sanuchem87@gmail.com)
}

MS received 6 June 2018; accepted 16 December 2018; published online 13 May 2019

\begin{abstract}
Capsicum extract at different concentrations $(0.5-3.0 \mathrm{ml})$ was blended with a chitosan polymer to obtain the composite films. It was compared with the pristine film to examine their antioxidant, mechanical, barrier, biodegradability, stability and antimicrobial properties. The morphology of the films was studied using a scanning electron microscope. From the obtained results, it was observed that the antioxidant, mechanical, biodegradability, stability and antimicrobial properties were enhanced when compared to the other films. The barrier properties of the composite films showed a decrease in activity when compared to the pure chitosan film. It may be due to the incorporation of a capsicum extract agent into the chitosan polymer matrix which plays a vital role in enhancing the overall properties.
\end{abstract}

Keywords. Biopolymer; chitosan; natural extract; capsicum; composite film.

\section{Introduction}

Food packaging is a vital part of modern society. The principal roles of food packaging are to protect food products from chemical, physical and biological influences [1]. However, synthetic polymer packaging causes many demerits like cost-effective, release of toxic gases and nonbiodegradability. To overcome these problems, biopolymers like chitosan, chitin, guar gum, starch and cellulose are being used. Chitosan is chemically defined as a copolymer of á- $(1,4)$ glucosamine $\left(\mathrm{C}_{6} \mathrm{H}_{11} \mathrm{O}_{4} \mathrm{~N}\right)_{n}$, having different numbers of $N$-acetyl groups [2]. It is the deacetylated form of chitin and it is found to be the second most polysaccharides after cellulose. Owing to its exceptional properties like biodegradability, non-toxicity and antimicrobial and antioxidant activities, it has been used as food preservative materials in many food industries [3]. Pepper (Capsicum annuum L.) is rich in vitamin $\mathrm{C}$ (ascorbic acid), pro-vitamin A (carotene) and calcium. The daily intake of $50-100 \mathrm{~g}$ fresh pepper fruits could provide $100 \%$ and about $60 \%$ of the recommended amounts of vitamin $\mathrm{C}$ and A, respectively. It possesses excellent antioxidant and anticarcinogenic properties [4]. In ancient times, it has been used as the food flavouring agent for human health [5]. The pungent chemical known as capsaicinoids is majorly present in capsicum. Capsaicin, dihydrocapsaicin, nordihydrocapsaicin, homocapsaicin and homodihydrocapsaicin are found to be the five natural capsaicinoids, but the profuse and effective are capsaicin and dihydrocapsaicin [6]. In this research, the properties of the capsicum extract blended chitosan composite films of different concentrations were prepared and compared with the pristine film for active food packaging application.

In the present work, $\mathrm{C}$ represents the pristine chitosan film, whereas C-0.5E, C-1.0E, C-1.5E, C-2.0E, C-2.5E and C-3.0E denote the different concentrations $(0.5,1.0,1.5,2.0,2.5$ and $3.0 \mathrm{ml}$ ) of natural extract blended chitosan composite films, respectively.

\section{Results and discussion}

The antioxidant activity, tensile strength, water vapour permeability (WVP), biodegradability and stability of the composite films are shown in table 1.

\subsection{Antioxidant activity of films}

The capsicum extract incorporated films showed enhanced antioxidant activity when compared to the pristine chitosan film (12\%). Since the capsaicin present here acts as an antioxidant agent in preventing lipid oxidation. As we increase the concentration of the capsicum extract from 0.5 to $2.5 \%$, the radical scavenging activity also increases from 19 to $59 \%$, respectively, whereas C-3.0E showed the contrast result. The enhancement may be due to the interaction between amide compounds present in the capsicum extract and the functional group of chitosan molecules that could influence the 2,2-diphenyl-1-picrylhydrazyl (DPPH) radicalscavenging ability of the films. 
Table 1. Antioxidant, tensile strength, WVP, biodegradability and stability of composite films.

\begin{tabular}{lccccc}
\hline Film types & $\begin{array}{c}\text { Antioxidant } \\
(\mathrm{RSA} \%)\end{array}$ & $\begin{array}{c}\text { Tensile strength } \\
(\mathrm{MPa})\end{array}$ & $\begin{array}{c}\text { WVP } \\
\left(\mathrm{mg} \mathrm{cm}^{-2} \mathrm{~min}^{-1}\right)\end{array}$ & $\begin{array}{c}\text { Biodegradability-soil } \\
\text { burial (30 days) } \\
\text { Weight loss (\%) }\end{array}$ & $\begin{array}{c}\text { Stability-buffer solution } \\
(21 \text { days }) \\
\text { Weight retained (\%) }\end{array}$ \\
\hline C & 12 & 28 & 1.75 & 76 & 71 \\
C-0.5E & 19 & 34 & 1.50 & 79 & 74 \\
C-1.0E & 25 & 40 & 1.25 & 84 & 78 \\
C-1.5E & 36 & 45 & 1.19 & 88 & 81 \\
C-2.0E & 47 & 50 & 0.97 & 92 & 83 \\
C-2.5E & $\mathbf{5 9}$ & $\mathbf{6 1}$ & $\mathbf{0 . 7 5}$ & $\mathbf{9 5}$ & 85 \\
C-3.0E & 42 & 55 & 0.84 & 93 & 85 \\
\hline
\end{tabular}

\subsection{Tensile strength of the films}

The tensile strength result showed that as the concentration of the capsicum extract increases, the tensile strength of the film also increases from 28 to $61 \mathrm{MPa}$. It may due to the hydrogen bonding between the amino functional group present in the chitosan and the capsaicin active group present in the capsicum extract. But further addition of the extract (3.0\%) to the polymer solution, the tensile strength tends to decrease due to some agglomeration of the extract on the chitosan film.

\subsection{WVP of the films}

The pure chitosan film showed a higher WVP value (1.75) because of its hydrophilic nature. The composite films namely, C-0.5E, C-1.0E, C-1.5E, C-2.0E and C-2.5E showed a decrease in values (from 1.50 to 0.75 ) as we increase the concentration of the capsicum extract, respectively, except for the C-3.0E film. It may be due to the hydrophobic nature of the capsaicin active group, which resists the inward bounding of water molecules from the atmosphere. Besides the good interaction of the two components, the presence of hydrogen bonds that are responsible for hydrogen bonding with water molecules will get reduced and result in a decrease in the WVP values.

\subsection{Biodegradability of the films}

The biodegradability test was carried out using the soil burial method for 30 days. It was found that as we increase the concentration of the capsicum extract, the biodegradability activity also increases and thus all the prepared films showed $>75 \%$ of weight loss in one month. It may be due to the presence of bioactive groups like capsaicin (contains the amide molecules) and $-\mathrm{NH}_{2}$ functional groups (chitosan) in the composite films. However, active groups present in the composite films will undergo an enhanced reaction by penetrating the layer of microorganisms present in the soil and result in the degradation process more effectively.

\subsection{Stability of the films}

The stability measurement of the prepared films was performed under physiological conditions at regular time intervals. From the obtained results, it was examined that on the 21 st day, the weight retained on each films was measured to be $71,74,78,81,83,88$ and $85 \%$ for C, C-0.5E, C-1.0E, C1.5E, C-2.0E, C-2.5E and C-3.0E films, respectively. Overall, all the prepared films showed $>70 \%$ of stability in maintaining weight in buffer solutions. In addition to this, the stability increases when we incorporate the natural extract into the chitosan film and further, on increasing the concentration of the capsicum extract, an increase in stability was found. It may be due to the healthy bonding between the functional groups present in the capsicum extract and the chitosan molecules.

\subsection{Scanning electron microscopy (SEM) of the films}

The morphology of the pristine chitosan film (C) and the optimized $2.5 \%$ capsicum extract incorporated chitosan film (C-2.5E) are shown in figure 1. The pure chitosan film shows a spongy surface, but the natural extract incorporated films show a rough surface. It may be due to the addition of the capsicum extract to the polymer matrix. From the obtained images, it was evident that the addition of the capsicum extract had played its role in the chitosan matrix.

\subsection{Antibacterial activity of the films}

The antibacterial activities of the chitosan film (C) and the optimized composite films $(\mathrm{C}-2.5 \mathrm{E})$ were tested against the Gram-negative (E. coli) and Gram-positive bacteria ( $S$. aureus) and are shown in figures $2 \mathrm{a}$ (positive control) and $2 \mathrm{~b}$ (negative control). It was observed that in both the cases, the optimized composite film showed more activity when compared to the pristine film. In addition to this, more antibacterial activity was found towards Gram-positive bacteria when compared to the Gram-negative bacteria. It may be due to the presence of the lipopolysaccharide layer (resist the entering 

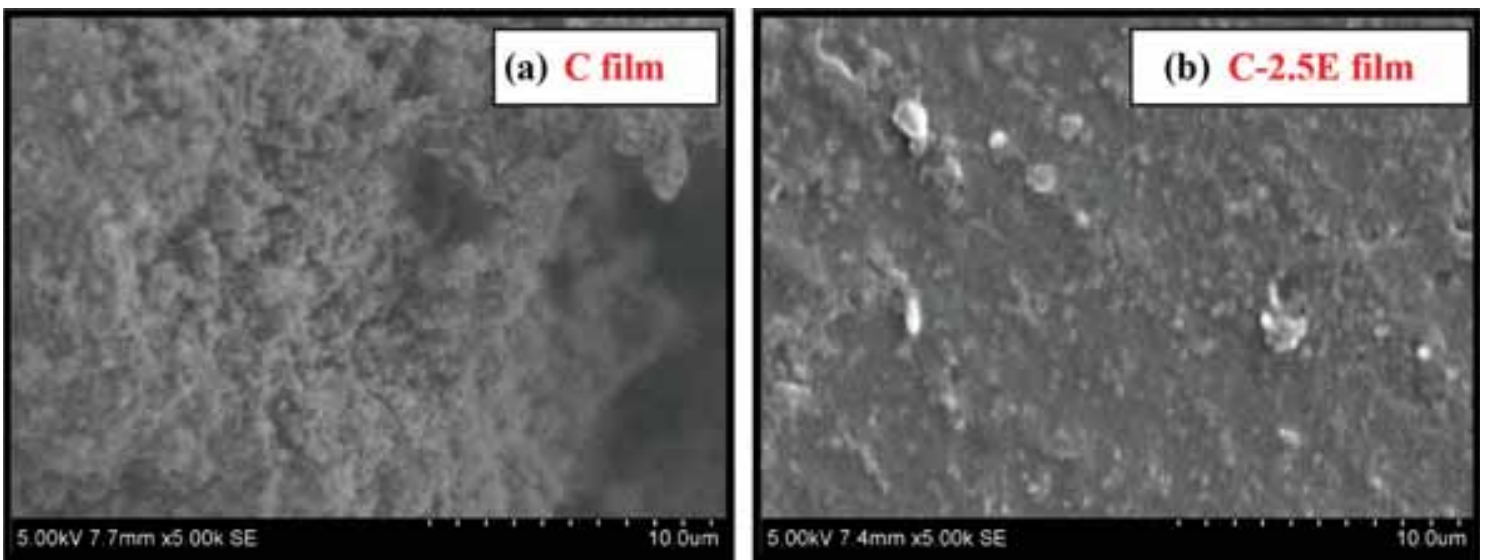

Figure 1. SEM images of (a) chitosan (C) and (b) composite (C-2.5E) films.

(a)

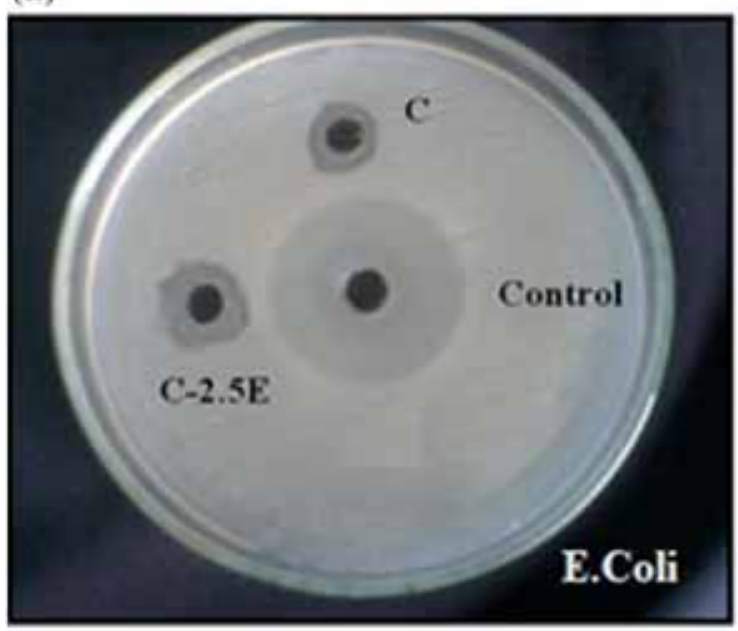

(b)

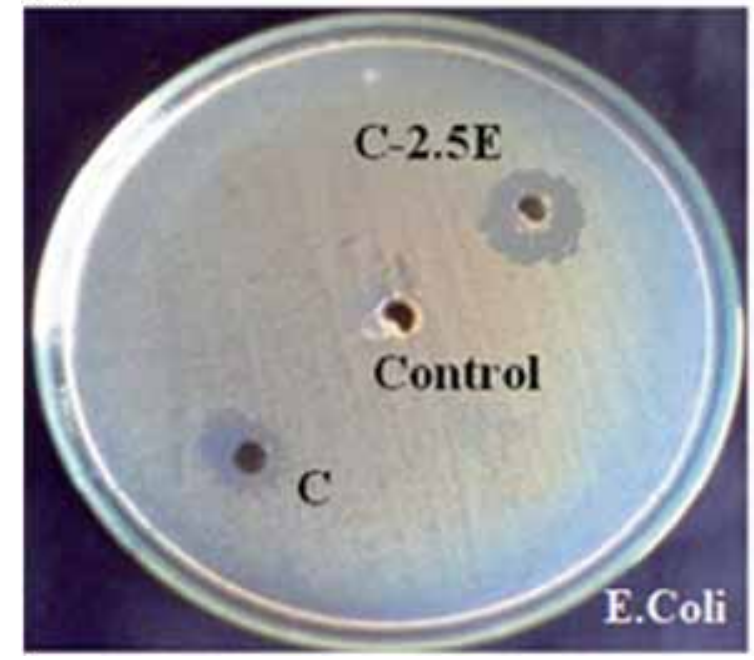

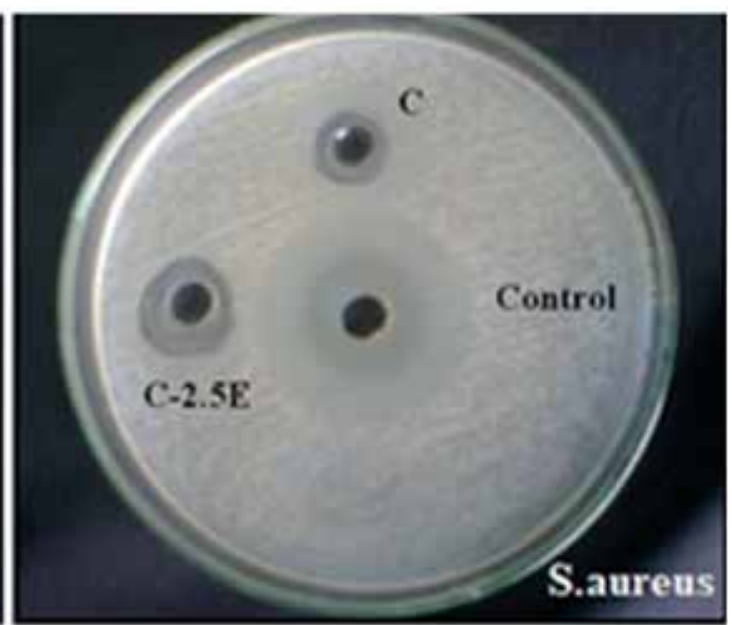

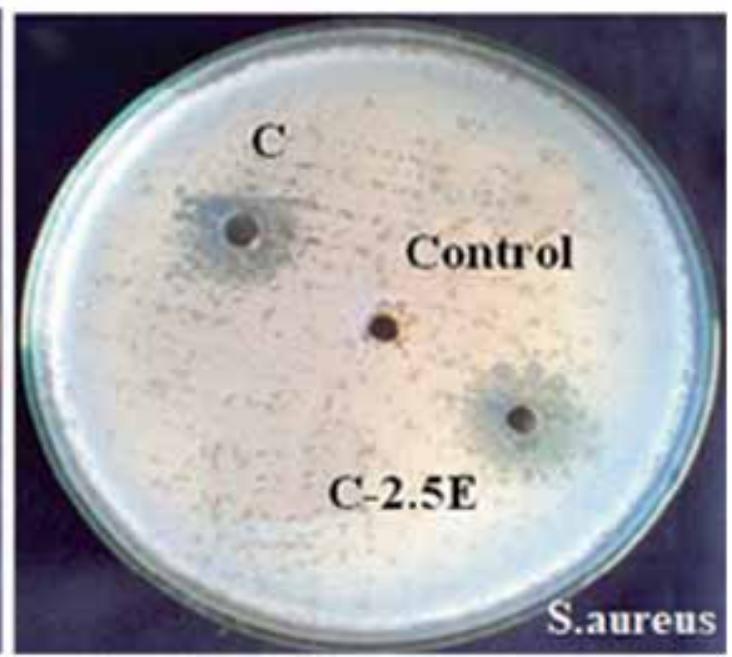

Figure 2. Antibacterial activity of the films $(\mathbf{a})$ : $(\mathrm{C}=$ chitosan, $\mathrm{C}-2.5 \mathrm{E}=2.5 \mathrm{ml}$ extract blended chitosan composite film, control = positive control (azithromycin) $)$ and $(\mathbf{b})(\mathrm{C}=$ chitosan, $\mathrm{C}-2.5 \mathrm{E}=2.5 \mathrm{ml}$ extract blended chitosan composite film, control $=$ negative control $(\mathrm{DMSO})$ ). 
of foreign substances) in the Gram-negative bacteria, while this was absent in the layer of Gram-positive bacteria.

\section{Materials and methods}

\subsection{Preparation of capsicum natural extract}

For the preparation of capsicum extract, $5 \mathrm{~g}$ of capsicum was chopped and ground in a blender well. Further, it was mixed with $250 \mathrm{ml}$ of distilled water and stirred while heating for $24 \mathrm{~h}$. Next day, the extracted solution was filtered and stored until use [7].

\subsection{Preparation of chitosan-capsicum composite films}

In the preparation of composite films, $1 \mathrm{~g}$ of chitosan was mixed with $1 \%$ of glacial acetic acid solution. To the prepared chitosan solution, different concentrations $(0.5,1.0,1.5,2.0$, 2.5 and $3.0 \mathrm{ml}$ ) of the capsicum extract were added along with few drops of glycerol to obtain the composite films by the solution cast method [8-11].

\subsection{Antioxidant activity}

Antioxidant activity was determined using the DPPH assay method. At $517 \mathrm{~nm}$ of absorption, the DPPH solution and sample were mixed and measured. The above step was repeated several times and the mixture was left in the dark at room temperature. Their absorption was monitored after a time interval of $20 \mathrm{~min}$ [12].

\subsection{Tensile strength}

The tensile strength was measured for the synthesized films and expressed in MPa using the Hounsfield Universal Testing Machine of $50 \mathrm{kN}$.

\section{$3.5 W V P$}

WVP was carried out using the WVP tester. The testing method is based on an aluminium oxide capacity sensor, which measures the relative humidity in the upper chamber and the lower chamber is saturated with water. The transmission of water from the lower to the upper chamber through the prepared films will give the transmission rate [13].

\subsection{Biodegradability activity}

The soil burial method was followed to test the biodegradability of the prepared composite films. The soil parameters such as temperature, moisture and $\mathrm{pH}$ were tested and found to be around $28-30^{\circ} \mathrm{C}, 65-75 \%$ and $6-7$, respectively. At a particular interval of time, the buried films were taken out, washed with water and dried at room temperature for $24 \mathrm{~h}$ [14].

\subsection{Stability of the films}

The stability measurement of the prepared chitosan composite films $(5 \times 5 \mathrm{~cm})$ was performed by incubating the films in the $\mathrm{pH}$ range of 7.4 in $5 \mathrm{ml}$ of phosphate buffered saline. All the samples were incubated on a shaker at $45 \mathrm{rpm}$ around $37^{\circ} \mathrm{C}$. At the regular time intervals $(3,6,9,12,15,18$ and 21 days), the films were taken out of the medium and washed with distilled water, dried in an oven $\left(45^{\circ} \mathrm{C}\right)$ and the weight of this films was measured. The stability was expressed as the accumulated weight retained by the films [15].

\subsection{SEM}

The VEGA3 TESCAN instrument with an accelerating voltage of 5-20 kV was used to study the morphology of all the synthesized materials.

\subsection{Antibacterial activity}

Antibacterial activities of the films were tested using the well diffusion method against Gram positive and Gram negative bacteria. The bacterial cultures were swabbed onto the sterile Petri-dish containing the nutrient agar (solidify). Wells with a diameter of $10 \mathrm{~mm}$ were drilled by using a cork borer. Later, $100 \mu 1$ concentration of the sample, one positive (azithromycin) and one negative control (dimethyl sulphoxide, DMSO) were swabbed. The plates were incubated for $24 \mathrm{~h}$, then the diameter of the inhibition was measured [16].

\section{Conclusion}

In summary, natural extract incorporated composite films were successfully synthesized using the solution cast method. From the obtained results, it was observed that there was an increase in antioxidant, mechanical, biodegradability, stability and antimicrobial activities and a decrease in WVP. It may be due to the presence of the capsicum natural extract in the chitosan matrix. Here, the capsaicin functional group interacts well with the amino functional group of the chitosan polymer and enhances the properties of the materials. In addition, the hydrophobic nature of the capsaicin group plays a vital role in resisting the incoming moisture to the composite film. In addition to this, the $\mathrm{C}-2.5 \mathrm{E}$ film was found to be the optimized film when compared to the other films. Therefore, these films are found to be an ideal material for active food packaging applications.

\section{Future work}

Further, the prepared films can be used to test the different kinds of food samples (vegetables and fruits) and in investigating the variations in the colony forming unit, nutrition content, etc., of the food materials that were treated using control films and composite films. 


\section{References}

[1] Kenneth M and Betty B 2007 J. Food Sci. 72 R39

[2] Shanta P, Paras N Y and Rameshwar A 2015 Nepal J. Sci. Tech. 1699

[3] Amalia C M, Elisabeta E T, Vlad I P and Mona E P 2015 Agro Life Sci. J. 452

[4] Rosa M M, Ana J, Paloma R, Félix R, Sierra B, Marina L et al 2013 Int. J. Mol. Sci. 149556

[5] Horacio B G, Luis L M, Esaú R S, Alfonso P G and Gabriel R L 2013 Chilean JAR 73329

[6] Alberto G Z, Erick S C, Guadalupe L O J, Rebeca P M, Juan Carlos R O and José L G H 2013 Molecules 18 13471

[7] Ajitha B, Ashok Kumar Reddy Y and Sreedhara Reddy P 2015 Mater. Sci. Eng. 49373
[8] Song H Y, Shin Y J and Song K B 2012 J. Food Eng. 113541

[9] Jessica I L N, Nancy P D Z, Carlos V S, José A M B, Ulises P G, Francisco P D et al 2018 Materials 11120

[10] Gaofeng Y, Hua L, Bingjie Y, Xiaoe C and Haiyan S 2015 Molecules 2011034

[11] Hongxia W, Jun Q and Fuyuan D 2018 J. Agric. Food Chem. 66 395

[12] Kim S J, Cho A R and Han J 2013 Food Control 29112

[13] Manikantan M R and Varadharaju N V 2012 Indian J. Eng. Mater. Sci. 1954

[14] Wang H, Wei D, Zheng A and Xiao H 2015 Polym. Degrad. Stabil. 11614

[15] Pourramezan Z, Kasra Kermanshahi R and Katbab A A 2017 Nanomed. Res. J. 2123

[16] Abdollahzadeh E, Rezaei M and Hosseini H 2014 Food Control 35177 